\title{
Semiclassical treatment of a Brownian ratchet using the quantum Smoluchowski equation
}

\author{
Liam Cleary, ${ }^{1}$ William T. Coffey, ${ }^{1}$ Yuri P. Kalmykov, ${ }^{2}$ and Serguey V. Titov ${ }^{3}$ \\ ${ }^{1}$ Department of Electronic and Electrical Engineering, Trinity College, Dublin 2, Ireland \\ ${ }^{2}$ Laboratoire de Mathématiques, Physique et Systèmes, Université de Perpignan, 52 Avenue de Paul Alduy, \\ 66860 Perpignan Cedex, France \\ ${ }^{3}$ Institute of Radio Engineering and Electronics, Russian Academy of Sciences, Vvedenskii Square 1, Fryazino 141190, Russia
}

(Received 22 July 2009; published 9 November 2009)

\begin{abstract}
Quantum effects in the noninertial Brownian motion of a particle in a one-dimensional ratchet potential are treated in the high temperature and weak bath-particle coupling limit by solving a quantum Smoluchowski equation for the time evolution of the Wigner function in configuration space. In particular, an analytical expression for the stationary average drift velocity for constant driving forces is presented including quantum corrections to any order in Planck's constant. The corresponding frequency response is determined using continued fractions in both the linear approximation holding for small ac driving amplitude and in the nonlinear regime for arbitrary driving amplitude exhibiting pronounced ac induced frequency dependence of the dc component of the average drift velocity. Moreover, Shapiro steps are apparent in the dc characteristics for strong ac driving just as in the dc current-voltage characteristics of a point Josephson junction.
\end{abstract}

DOI: 10.1103/PhysRevE.80.051106

PACS number(s): 05.40.Jc, 05.60.Gg, 05.70.Ln

\section{INTRODUCTION}

The rectified current of Brownian particles arising from the combination of an asymmetric, spatially periodic ratchet potential with an unbiased, undulating stimulus coupled to a thermal bath has long been of interest, both as an example of a nonequilibrium system and as a microscopic transport device since it effectively represents a mechanical diode $[1,2]$. The abiding interest in forced thermal ratchets is reflected in the diversity of papers [1-5] dealing with their transport properties. In general, such ratchets are characterized by periodic potentials with broken spatial symmetry, affording the possibility of extracting a net particle flow from unbiased (i.e., vanishing on time averaging) driving. The ratchet effect has been reported experimentally in a variety of physical and biological systems, which are so small that the thermal noise cannot be ignored, because the energy barriers to thermal activation are just a few $k T$, unlike in the macroscopic world, where the barriers are effectively infinite [1]. Ratchet effects have also been widely used to explain the action of motor proteins and to suggest novel nanomachines, e.g., Brownian motors, operating far from thermal equilibrium by extracting energy fluctuations to work against external loads [6].

Until comparatively recently, the ratchet system has been studied in the classical limit. Here, the dynamics are governed by the Langevin equation [7]. This equation is simply the Newtonian equation of motion of the particle in the ratchet potential driven by external forces, augmented by stochastic terms describing the interaction with the surrounding heat bath so that the position and momentum of the particle now become random variables. The Langevin or stochastic differential equation is also accompanied by a Fokker-Planck equation [8] describing the time evolution of the joint probability density function $W(x, p, t)$ of the representative points (positions $x$ and momenta $p$ ) of the particle in phase space $(x, p)$. The representative points are of course the realizations or the infinite set of random phase-space trajectories generated by the Langevin equation. In applications to the trans- lational motion of a point particle, where the Hamiltonian of the closed system is separable and additive, the FokkerPlanck equation in the phase space of positions and momenta is known as the Klein-Kramers equation [8].

The Klein-Kramers equation is usually solved by expanding the momentum part of the joint phase-space distribution $W(x, p, t)$ in orthogonal Hermite polynomials of order $n$, namely, $H_{n}(p)$, where $n=0,1,2 \ldots$, leading to a partial differential-recurrence relation for the separation coefficients $\varphi_{n}(x, t)$ in configuration space $x$ [9]. Expansion of the distribution function in Hermite polynomials is equivalent to taking the Fourier transform over the momentum distribution, i.e., calculating the characteristic function of the momentum [10]. The separation coefficient $\varphi_{0}(x, t)$ is of particular interest as it constitutes the exact configuration space distribution function. The partial differential-recurrence relation in $n$ generates an infinite hierarchy of partial differential-recurrence equations for the configuration space functions, known as Brinkman's hierarchy $[9,11]$. These may be solved recursively in the frequency domain $(s)$ as a continued fraction of current operators in the coordinate representation or their equivalent Heisenberg matrices (see Appendix C of Ref. [9]). For a periodic potential, such as the ratchet however, the $\varphi_{n}(x, t)$ may be further expanded in a spatial Fourier series reducing the problem to the solution of a set of ordinary differential-recurrence relations in the order $n$ of the Hermite polynomials and $q$ the number of harmonics of the Fourier series. The hierarchy of recurrence relations in the recurring numbers $n, q$ ultimately yields the time behavior of the decay functions of the system when it is solved by matrix continued fraction methods in the frequency domain, having first converted it to a matrix three-term differential-recurrence relation in $n$. The same hierarchy of ordinary differentialrecurrence equations may be generated by averaging the Langevin equation over its realizations $\{x(t), p(t)\}$ at a time $t$ in phase space given a point set of initial values $\left\{x\left(t_{0}\right), p\left(t_{0}\right)\right\}$, as described in Ref. [7]. The averaging over the realizations for the point or sharp set of initial values ultimately yields the Green's function or transition probability for the system. 
The solution for other initial conditions, e.g., a MaxwellBoltzmann distribution, may be found by taking an average of the time-dependent solution for sharp initial conditions over that distribution.

Now, the configuration space distribution function $\varphi_{0}(x, t)$ and any averages over it include exactly the inertia of the particles, which in the context of the Brownian motion is simply another way of stating that the results (e.g., escape rates) are valid for all ranges of the bath-particle dissipation as treated classically. The calculations may however be considerably simplified in the noninertial limit, where the dissipative term in the Langevin equation dominates the inertial term. This amounts to the ansatz that a Maxwellian distribution of the momenta has set in well before the steady state distribution of the displacements has been attained. In other words, the momentum is a fast variable while the position is a slow variable. The simplification occurs because in the noninertial limit the coordinate representation [9] of the solution of the hierarchy of recurrence relations for the Laplace transform $\widetilde{\varphi}_{n}(x, s)$ yields, on inversion to the time domain, a second-order partial differential equation for the configuration space distribution function $\varphi_{0}(x, t)$, namely, the Smoluchowski equation. This equation replaces the inverse Laplace transform of the continued fraction of current operators occurring in the inertial case which represents an $n$th order partial differential equation and is consequently difficult to solve. In the case of periodic potentials, expansion of the solution of the Smoluchowski equation in a spatial Fourier series then leads to an ordinary differential-recurrence relation, recurring only in the order $q$ of the series. For the resistively shunted junction model (RSJ-model) of the Josephson junction closely related to the ratchet, where the capacitance is ignored (corresponding to neglecting the inertia in the mechanical analog), this recurrence relation reduces to a three-term one. Hence, it may be solved in the frequency domain using scalar continued fractions yielding the impedance of the junction. In particular, the time-independent solution yields the dc current-voltage characteristics [7]. For the ratchet potential, however, the scalar recurrence relation does not in general reduce to a three-term one, rather it reduces only to a matrix three-term recurrence relation. Hence, in order to solve it matrix continued fractions must again be used, albeit these are considerably simpler than those associated with the exact inertial solution as typically only finite matrices are involved rather than the infinite matrices associated with the order $n$ of the Hermite polynomials of the inertial solution.

In tandem with the study of classical Brownian ratchets, interest has also focused on extending the ratchet to the quantum regime, particularly in the context of molecularsized physical engines, where it appears that transport properties such as the average drift velocity are considerably modified in relation to their classical counterparts [12]. Hitherto, quantum ratchets have usually been investigated in the context of established devices such as the Josephson junction and a few novel devices such as rocked electron ratchets [13-16]. A convenient starting point for our discussion is therefore the analysis of the Josephson junction by Zwerger [17]. Following an earlier paper on the quantum Brownian motion in a periodic potential [18], he used the influence- functional formalism developed by Feynman and Vernon [19] combined with the system-plus-bath Hamiltonian of Caldeira and Leggett [20] to investigate quantum effects in the dc current-voltage characteristic of a small Josephson junction. The Caldeira-Leggett Hamiltonian was originally used to study the macroscopic quantum tunneling of the phase difference across a Josephson element in an RFSQUID ring [20]. This Hamiltonian couples a (tagged) particle linearly to a bath of harmonic oscillators, e.g., a string or transmission line at temperature $T$, which simultaneously provides both a frictional and a fluctuating force for the particle and lends itself to quantization.

The path-integral approach above to both the Josephson junction and the ratchet has, however, the disadvantage that it is relatively difficult to visualize in terms of the classical representation as averages of dynamical quantities over phase-space distributions. An answer is provided by Wigner's phase-space representation of quantum mechanics in terms of a certain Fourier transform (prompted by the symmetries of the Heisenberg-Weyl group of translations) of the density matrix [21], so generating a quasiprobability distribution in phase space. This distribution corresponds to the sum of the diagonal elements of the density matrix and is called the Wigner function. Here, the $c$-numbers $x, p$ represent the corresponding position and momentum quantum operators. The Wigner function evolves according to a quantum analog of the Liouville equation of classical statistical mechanics, which may be solved at high temperatures by means of perturbation theory in a quantum parameter $\Lambda$ $=\hbar^{2} \beta^{2} /(24 m)$, where $\hbar$ is Planck's reduced constant, $\beta$ $=(k T)^{-1}$ is the fugacity and $m$ is the mass of the particle. The Wigner function has most of the attributes of a true phasespace distribution function [22] (for illuminating discussions see the chapters by Baker and Feynman in Ref. [23]). Moreover, since it is usually determined explicitly via a high temperature perturbation expansion the Wigner function is eminently suited to the study of quantum corrections to classical distributions, i.e., the semiclassical limit. Indeed, Wigner's representation of quantum mechanics (rather than the conventional representations as the probability densities of the position or momentum, which are simply the marginal probability density functions of the Wigner distribution) now known as the Wigner-Moyal quantization [24], was originally given for closed thermodynamic systems, the ultimate purpose being to obtain high temperature quantum corrections to classical thermodynamic equilibrium. Essentially, Wigner's ideas comprise the extension of classical methods to the quantum domain. Thus quantum mechanical observables, expressed as the trace of the product of the relevant operator and the density matrix, may be calculated [24] via the Weyl symbol of that operator by averaging the corresponding classical quantity ( $c$-number) over a quasiprobability distribution in phase space. The Wigner representation, which is simply a particular example of a representation distribution for systems with symmetries governed by the Heisenberg-Weyl group [25] alluded to above was later generalized by Stratonovich to all representation distributions, which have a classical meaning. Examples are those associated with the $\mathrm{SU}(2)$ rotation group [25]. This approach has very recently been extended to magnetic spins by Kalmykov et al. [26]. 
As far as the quantum Brownian motion is concerned, which involves extending the Wigner representation to open systems, the necessary phase-space formalism has been established by Coffey et al. [9]. They have shown how to obtain $c$-number master equations, akin to the Fokker-Planck equation (FPE), for the evolution of a time-dependent quasiprobability distribution (Wigner function) in phase space incorporating the bath-particle interaction, which describe high temperature quantum effects. These master equations, characterized (unlike the FPE) by Kramers-Moyal coefficients [8] depending on both the derivatives of the potential and on the momentum, are derived by imposing the Wigner distribution of the closed system as the equilibrium distribution. The foregoing ansatz has been vindicated by Dillenschneider and Lutz [27] for the particular case of the harmonic oscillator potential. They have shown quantitatively that it corresponds to neglecting small terms in the square of the bath-particle interaction in the equilibrium distribution of the open system, which is ultimately the meaning of the term weak interaction in the present context.

Now, the master equations lead to quantum analogs of the Brinkman equations in configuration space which may be solved on applying perturbation theory in the quantum parameter $\Lambda$ by continued fraction methods in the frequency domain in substantially the same way as the Klein-Kramers equation. Moreover, in the noninertial limit the quantum Brinkman equations yield just as the classical case a generalization of the Smoluchowski equation for the configuration space quasiprobability density function in which quantum effects appear in the diffusion coefficient, while the drift coefficient remains unchanged. This equation is similar but not identical to a quantum Smoluchowski equation originally obtained by Ankerhold et al. [28] from the path-integral formulation of quantum mechanics, insofar as in the latter Smoluchowski equation both the drift and the diffusion coefficients are altered by quantum effects. However, in subsequent publications $[27,29]$ and in recent books $[30,31]$ it has been accepted that the equation proposed by Ankerhold et al. [28] should read the same as that of Coffey et al. [9]. In other words, quantum effects should appear in the diffusion coefficient alone.

It is the purpose of this paper to demonstrate how the quantum Smoluchowski equation, derived by extending Wigner's formulation of quantum mechanics to open systems with the stosszahlansatz of Brownian motion [9], may be used to study high temperature quantum corrections to the average drift velocity of the Brownian ratchet in the noninertial limit as previously accomplished for the Josephson junction [32,33]. We shall present the dc average drift velocity both in closed integral form and as an easily computed continued fraction. Proceeding to the frequency dependent stationary case, we shall investigate quantum effects in the frequency dependence of the ac average drift velocity. We shall consider these effects in both the linear response, valid for small driving amplitudes, and in the nonlinear response valid for arbitrary driving amplitude. Furthermore, we shall demonstrate that the nonlinear response exhibits pronounced frequency dependence of the dc average drift velocity as well as Shapiro-like steps in the curve of that quantity versus dc bias just as the dc current-voltage characteristic of the point
Josephson junction for strong driving current. In determining the various solutions, we shall describe the alterations which must be made to the continued fraction as one proceeds from the stationary state to the linear response and finally to the nonlinear response. An attractive feature is the similarity of the fraction for each of the three solutions so that only slight alterations are required in each case.

\section{QUANTUM SMOLUCHOWSKI EQUATION FOR THE RATCHET POTENTIAL}

The master equation for the time evolution of the Wigner distribution $W(x, p, t)$ in the phase space of positions $x$ and momenta $p$ of a particle of mass $m$ moving along the $x$ axis in a potential $V(x)$ is

$$
\begin{aligned}
\frac{\partial W}{\partial t} & +\frac{p}{m} \frac{\partial W}{\partial x}-\frac{1}{i \hbar}\left[V\left(x+\frac{i \hbar}{2} \frac{\partial}{\partial p}\right)-V\left(x-\frac{i \hbar}{2} \frac{\partial}{\partial p}\right)\right] W \\
& =\hat{M}_{D} W
\end{aligned}
$$

where $\hat{M}_{D}$ is the collision kernel operator. The left-hand side is simply the quantum analog of the classical Liouville equation, so that setting the right hand side equal to zero, Eq. (1) constitutes the evolution (continuity) equation for the Wigner distribution function in the phase space of the closed system as originally given by Wigner [21]. He solved this equation via perturbation theory in $\Lambda$ in order to obtain semiclassical corrections to the Maxwell-Boltzmann distribution. Hence, classical transition state theory is modified because quantum effects due to high temperature tunneling near the top of the barrier lower the effective barrier heights [34]. The righthand side of Eq. (1) contains the collision kernel $\hat{M}_{D}$, responsible for the bath-particle interaction, here the Brownian motion of the open system. By representing this kernel as a Kramers-Moyal [8] expansion truncated at the second term as in the classical Brownian motion and assuming frequencyindependent damping one may calculate by imposing the Wigner distribution of the closed system as the first approximation to the equilibrium distribution the Kramers-Moyal coefficients to any power in $\hbar$, thus obtaining the explicit form of the collision kernel, which to second order in $\hbar$ is [9]

$$
\begin{aligned}
\hat{M}_{D} W= & \gamma \frac{\partial}{\partial p}\left[p W+\frac{m}{\beta}\left\{1+2 \Lambda V^{\prime \prime}-\frac{2}{5} \Lambda^{2}\left[6 V^{\prime \prime \prime} V^{\prime}+2\left(V^{\prime \prime}\right)^{2}\right.\right.\right. \\
& \left.\left.\left.+3 V^{(4)}\left(\frac{p^{2}}{m}-\frac{5}{\beta}\right)\right]\right\} \frac{\partial W}{\partial p}\right]+\cdots
\end{aligned}
$$

Here, $\gamma=\zeta / m$ is a dissipation (damping) parameter characterizing the bath-particle interaction where $\zeta$ is the friction coefficient. In a quantum mechanical sense, Eq. (1) is valid in the high temperature and weak coupling limits, i.e., the correlation time characterizing the heat bath is so short that one can regard the stochastic process originating in the bath as Markovian. Furthermore, recalling that the equilibrium distribution of the open system may in general depend on the bath-particle interaction [35], the imposition of the Wigner distribution of the closed system as the equilibrium distribution is tantamount to neglecting terms of $o\left(\gamma^{2}\right)$ in the equi- 
librium distribution of the open system as has been formally demonstrated for the harmonic oscillator [27].

Now, either integration of Eq. (1) over $p$ using the explicit form of $\hat{M}_{D}$ Eq. (2) and proceeding to the noninertial limit just as Klein and Kramers [9,36] or considering the noninertial limit of the quantum Brinkman hierarchy [9,11] generated by expanding the momentum dependence of $W(x, p, t)$ in Hermite polynomials, then leads to the quantum Smoluchowski equation for the quasiprobability distribution function $P(x, t)=\int_{-\infty}^{\infty} W(x, p, t) d p$ in configuration space, namely,

$$
\frac{\partial P}{\partial t}=\frac{\partial}{\partial x}\left\{\frac{P}{\zeta} \frac{\partial V}{\partial x}+\frac{\partial}{\partial x}(D P)\right\},
$$

where $D(x)$ is the diffusion coefficient given by

$$
\begin{aligned}
D(x)= & \frac{1}{\zeta \beta}\left(1+2 \Lambda V^{\prime \prime}(x)-\frac{4 \Lambda^{2}}{5}\left\{\left[V^{\prime \prime}(x)\right]^{2}+3 V^{\prime}(x) V^{(3)}(x)\right.\right. \\
& \left.\left.-3 \beta^{-1} V^{(4)}(x)\right\}+\cdots\right),
\end{aligned}
$$

while the drift coefficient $-V^{\prime}(x) / \zeta$ remains as in the classical case so that an effective potential is not involved. The corresponding Langevin equation in the Stratonovich interpretation [37] reads

$$
\dot{x}(t)=-\frac{1}{\zeta} \partial_{x}\left\{V[x(t)]+\frac{\zeta}{2} D[x(t)]\right\}+\sqrt{\frac{\beta}{\zeta} D[x(t)] \lambda}(t),
$$

where the dot denotes the time derivative, and $\lambda(t)$ is a random force with the Gaussian white-noise properties

$$
\overline{\lambda(t)}=0, \quad \overline{\lambda(t) \lambda\left(t^{\prime}\right)}=(2 \zeta / \beta) \delta\left(t-t^{\prime}\right) .
$$

Here, the overbar means the statistical average over the realizations of the random force. Equation (5) may be used as an approximate description of the kinetics of a quantum Brownian particle in the noninertial limit. One may average Eq. (5) over its realizations in configuration space to obtain the averaged equation of motion

$$
\overline{\dot{x}}=\zeta^{-1} \overline{\partial_{x} V(x)}
$$

and thus the drift coefficient, coinciding with its classical counterpart. Now, since the quasiprobability distribution $P(x, t)[$ cf. Eq. (3)] is simply the trace of the density matrix operator $\hat{\rho}$, i.e., $P(x, t)=\langle x|\hat{\rho}| x\rangle$, one may calculate quantum mechanical expectation values just as classical ones, via the Weyl correspondence,

$$
\langle\hat{A}\rangle=\operatorname{tr}(\hat{\rho} \hat{A})=\int A(x) P(x, t) d x,
$$

so allowing one to study the influence of thermal as well as quantum mechanical fluctuations. In addition to this classical-like representation of expectation values, a further advantage of the semiclassical approach is that the classical definitions of work and heat remain valid allowing direct extension of fluctuation-dissipation theorems to quantum systems yielding important insights in the semiclassical limit [38,39].
We now specialize Eq. (3) to the quantum Brownian motion of a particle in a ratchet potential driven by a constant force $a$. We consider the one-dimensional, spatially periodic, $V(x)=V(x+L)$, asymmetric potential

$$
V(x)=V_{0}\left[\sin (2 \pi x / L)+b_{1} \sin (4 \pi x / L)+b_{2} \sin (6 \pi x / L)\right],
$$

where the constant $V_{0}$ determines the barrier height and the parameters $b_{1}$ and $b_{2}$ characterize the spatial asymmetry. Introducing the dimensionless variables

$$
\begin{gathered}
2 \pi x / L \rightarrow x, \quad t / \tau \rightarrow t, \quad \tau=\zeta \beta L^{2} / 4 \pi^{2}, \\
a \beta L / 2 \pi \rightarrow a, \quad 8 \pi^{2} \Lambda / \beta L^{2} \rightarrow \Lambda, \\
\beta V(x) \rightarrow V(x), \quad \beta V_{0} \rightarrow V_{0},
\end{gathered}
$$

the quantum Smoluchowski Eq. (3) then becomes

$$
\frac{\partial}{\partial t} P(x, t)=\frac{\partial}{\partial x}\left\{\left[V^{\prime}(x)-a\right] P(x, t)+\frac{\partial}{\partial x}[D(x) P(x, t)]\right\},
$$

where

$$
V(x)=V_{0}\left[\sin x+b_{1} \sin 2 x+b_{2} \sin 3 x\right]
$$

and the dimensionless diffusion coefficient is

$$
\begin{aligned}
D(x)= & +\Lambda V^{\prime \prime}(x)-\frac{\Lambda^{2}}{5}\left\{V^{\prime \prime}(x)^{2}+3\left[V^{\prime}(x)-a\right] V^{(3)}(x)\right. \\
& \left.-3 V^{(4)}(x)\right\}+\cdots .
\end{aligned}
$$

In passing, we remark that due to the mathematical difficulties involved relatively little attention has been paid to ac driving forces, where the time-dependent Smoluchowski equation must be used. In contrast, Brownian-ratchet dynamics have been studied extensively using the time-independent Smoluchowski equation. For example, current reversals via manipulation of the ratchet profile have been studied classically for a two-state Markovian driving force, where the governing master equation consists of two coupled Smoluchowski equations [40]. Similarly, the role of quantum tunneling and quantum reflection in the enhancement or suppression of the stationary average drift velocity of a quantum Brownian particle has been studied by Machura et al. [12] to first order in $\Lambda$ using the quantum Smoluchowski equation of Ankerhold et al. [28]. We note that they regard the diffusion coefficient in that equation as the leading term in the Taylor expansion of $\left[1-\Lambda V^{\prime \prime}(x)\right]^{-1}$ in order to exclude Maxwelldemon effects. Such an assumption is unnecessary here as the Wigner approach automatically furnishes the desired expansion of the diffusion coefficient in powers of $\Lambda$ [cf. Eq. (12)].

In order to lay the groundwork for the study of the timedependent case, pertaining to the stationary ac response, we must first consider the time-independent solution of the quantum Smoluchowski Eq. (10), which having been solved by quadratures will then be used to calculate the average drift velocity in the slow-switching limit of a symmetric two state 
driving force. We shall also present the average drift velocity in the form of a continued fraction.

\section{STATIONARY AVERAGE DRIFT VELOCITY}

The dimensionless quantum Smoluchowski equation in the stationary state governing the time-independent quantum Brownian motion of a particle in the ratchet potential with a constant tilt of arbitrary amplitude $a$ is

$$
\frac{\partial}{\partial x}\left\{\left[V^{\prime}(x)-a\right] P_{s t}(x)+\frac{\partial}{\partial x}\left[D(x) P_{s t}(x)\right]\right\}=0 .
$$

Under stationary conditions, the probability current satisfying the continuity equation

$$
\partial_{t} P(x, t)=-\partial_{x} J(x, t),
$$

must have the constant value $J(a)$, with the argument $a$ indicating that the current has its origin purely in the tilt. Hence, from Eq. (13) the constant probability current is

$$
J(a)=-\left[V^{\prime}(x)-a\right] P_{s t}(x)-\frac{\partial}{\partial x}\left[D(x) P_{s t}(x)\right] .
$$

The stationary average drift velocity of the particle $\langle\dot{x}\rangle[\mathrm{cf}$. Eq. (6) with $V^{\prime}(x)$ replaced by $\left.V^{\prime}(x)-a\right]$ is found by averaging the drift terms so that [8]

$$
\langle\dot{x}\rangle=-\int_{0}^{2 \pi}\left[V^{\prime}(x)-a\right] P_{s t}(x) d x,
$$

or, noting Eq. (14), in terms of the probability current

$$
\langle\dot{x}\rangle=\int_{0}^{2 \pi}\left\{J(a)+\frac{\partial}{\partial x}\left[D(x) P_{s t}(x)\right]\right\} d x .
$$

Since $P_{s t}(x)$ is bounded and therefore periodic [8], so that $P_{s t}(x+2 \pi)=P_{s t}(x)$, we ultimately have

$$
\langle\dot{x}\rangle=\int_{0}^{2 \pi} J(a) d x=2 \pi J(a) .
$$

Now, $P_{s t}(x)$ is normalized, i.e., $\int_{0}^{2 \pi} P_{s t}(x) d x=1$, so that following the arguments used in Ref. [8] (see Ch. 11) for the classical case we have by quadratures the analytical result for the tilt induced probability current

$$
J(a)=\frac{1-e^{-2 \pi a}}{\int_{0}^{2 \pi} D(x)^{-1} e^{-\Psi(x)} \int_{x}^{x+2 \pi} e^{\Psi(y)} d y d x},
$$

where

$$
\Psi(x)=\int^{x} D(y)^{-1}\left[V^{\prime}(y)-a\right] d y .
$$

Equation (18) was essentially derived by Kramers [36] in the context of the classical Smoluchowski equation (see also Ref. [41]) and extended to a tilted cosine potential by Risken [8]. In like manner, the stationary probability distribution may be determined from Eq. (14). We thus have the bounded distribution [8]

$$
P_{s t}(x)=D(x)^{-1} \frac{J(a) e^{-\Psi(x)}}{1-e^{-2 \pi a}} \int_{x}^{x+2 \pi} e^{\Psi(y)} d y,
$$

where the probability current $J(a)$ serves as a normalizing constant, analogous to the inverse partition function, $Z^{-1}$. In the case of zero tilt $(a=0)$, where the probability current is zero, the stationary solution is simply the Wigner equilibrium distribution in configuration space, which to first order in $\Lambda$ is [9]

$$
P_{s t}(x)=Z^{-1} e^{-V(x)}\left\{1+\frac{\Lambda}{2}\left[V^{\prime}(x)^{2}-2 V^{\prime \prime}(x)\right]+\cdots\right\},
$$

where $Z=\int_{0}^{2 \pi} P_{s t}(x) d x$ is the partition function. In the original uncorrected form of the quantum Smoluchowski equation proposed by Ankerhold et al. $[28,29]$ in which quantum effects also appear in the drift coefficient, the stationary distribution differs from the Wigner distribution (21) as emphasized in Ref. [32].

Equations (17)-(19) constitute the exact, analytical solution for the stationary average drift velocity in a potential $V(x)$ with constant driving force $a$. For a negative driving force one finds that $\langle\dot{x}\rangle_{-}(a)=\langle\dot{x}\rangle_{+}(-a)$ (the subscripts denote the separate cases of positive and negative amplitude). We may thus obtain an expression for the average drift velocity in the slow-switching limit of a symmetric, two-state, $\{+a$, $-a\}$ driving force, namely,

$$
\langle\dot{x}\rangle_{s s l}=\left[\langle\dot{x}\rangle_{+}(a)+\langle\dot{x}\rangle_{+}(-a)\right]=2 \pi[J(a)+J(-a)] .
$$

We remark that quantum effects in the Brownian ratchet have been studied in this switching regime, both in the noninertial case using the original quantum Smoluchowski equation of Ankerhold et al. [28] and in the inertial case via the Caldeira-Leggett master equation in phase space [13]. The right hand side of the Caldeira-Leggett master equation is often taken to be the same [42] as that of the Fokker-Planck equation. Thus quantum effects appear in the Liouville term only, leading in the noninertial limit to the classical Smoluchowski equation [unlike in the phase-space master Eq. (1), where the Kramers-Moyal coefficients of the kernel (2) contain both the momentum and the derivatives of the potential, ultimately leading in the noninertial limit to the quantum Smoluchowski Eq. (3)]. Now, in the noninertial case, we reiterate that adopting the equation of Ankerhold et al.[28], Machura et al. [12] regard the diffusion coefficient in that equation as the leading term in the Taylor expansion of [1 $\left.-\Lambda V^{\prime \prime}(x)\right]^{-1}$ in order that the second law of thermodynamics is not violated (see the inset of Fig. 1 of Ref. [12]). Furthermore, quantum noise enters in two places, via an effective potential and via an effective diffusion coefficient defined using the Taylor series expansion above. In contrast, in the quantum Smoluchowski equation used here the potential is the same as the classical case, while the expansion of the diffusion coefficient (12) in powers of $\Lambda$ is a consequence of the perturbation theory solution. Thus, the unphysical net particle current at zero tilt is automatically eliminated. This is exemplified by the graph of the stationary average drift velocity in the slow-switching limit $\langle\dot{x}\rangle_{s s l}$ vs the amplitude of the two-state driving force (tilt) $a$, which we plot in Fig. 1 for 


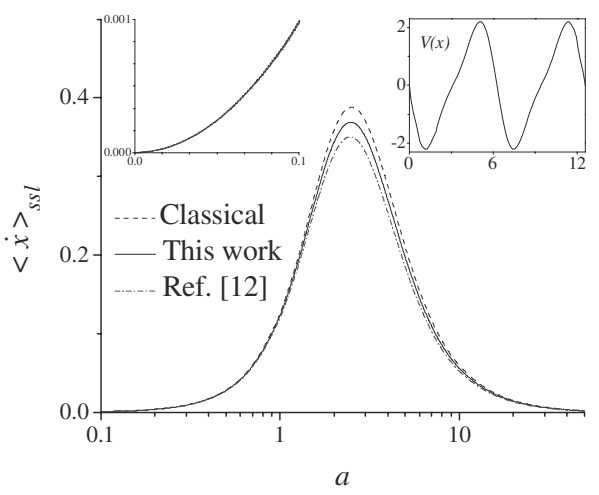

FIG. 1. The average drift velocity in the slow-switching limit $\langle\dot{x}\rangle_{s s l}$ vs two-state driving force $a$ for the ratchet potential $V_{0}=-2$, $b_{1}=0.25$, and $b_{2}=0$ (see right inset). The quantum case $(\Lambda=0.02)$ for the quantum Smoluchowski Eq. (13) and that of Ref. [12] is shown along with the classical case $(\Lambda=0)$. In all cases there is zero probability current (no Maxwell-demon behavior) at zero tilt (see left inset).

the ratchet potential $V_{0}=-2, b_{1}=0.25$ and $b_{2}=0$ (see right inset) by means of Eqs. (17)-(19). We include for comparison both the classical result $(\Lambda=0)$ and the prediction of Machura et al. [12] to first order in $\Lambda$ only. It is immediately apparent that besides eliminating the undesirable Maxwelldemon behavior (see left inset) the quantum Smoluchowski equation used here results in a smaller reduction in the average drift velocity due to quantum effects than that of Ref. [12]. By way of illustration of the convergence of the perturbation theory in $\Lambda$, we show in Fig. 2 for the ratchet potential $V_{0}=1, b_{1}=0.5$, and $b_{2}=0.5$, in which the harmonics are relatively prominent (see right inset) the dependence of the average drift velocity $\langle\dot{x}\rangle_{s s}$ on the two state driving amplitude $a$

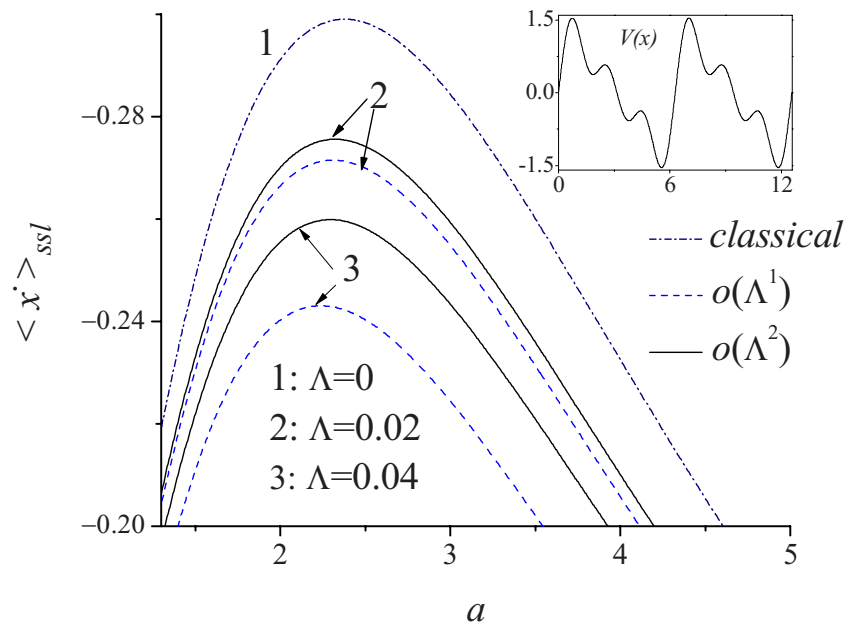

FIG. 2. (Color online) The average drift velocity in the low switching limit $\langle\dot{x}\rangle_{s s l}$ vs two-state driving force $a$ in the ratchet potential $V_{0}=1, b_{1}=0.5$, and $b_{2}=0.5$ (see inset) for various values of the quantum parameter, $\Lambda=0$ (curve 1 , classical limit), $\Lambda=0.02$ (curves 2) and $\Lambda=0.04$ (curves 3 ). Dashed and solid lines are the predictions of the quantum Smoluchowski equation to first- and second-order quantum corrections, respectively. Clearly as $\Lambda$ increases the higher order terms in the diffusion coefficient must be included. for various values of the quantum parameter, $\Lambda=0$ (curve 1, classical limit), $\Lambda=0.02$ (curves 2) and $\Lambda=0.04$ (curves 3 ). Clearly as $\Lambda$ increases the higher order terms in the diffusion coefficient must be included in order to achieve satisfactory convergence.

We have presented the stationary solution as obtained in integral form by quadratures. However, that solution may also be obtained via Fourier's theorem using continued fractions, which are particularly suited to the numerical evaluation of the Fourier coefficients. Moreover, the average drift velocity may be expressed as a linear combination of these coefficients. We remark that continued fractions are an efficient method of obtaining the exact stationary solution for ac driving forces and have the merit that only minor alterations are required in proceeding from the time-independent stationary solution to the ac response. Considering the timeindependent solution we make the Fourier expansion

$$
P_{s t}(x)=\frac{1}{2 \pi} \sum_{n=-\infty}^{\infty} C_{n} e^{i n x},
$$

where the time-independent coefficients (characteristic function of the random variable $x$ ) are

$$
C_{n}=\int_{0}^{2 \pi} e^{-i n x} P_{s t}(x) d x=\left\langle e^{-i n x}\right\rangle_{0},
$$

and the zero subscript denotes averaging over the stationary distribution $P_{s t}(x)$. For ease of presentation, we write the continued fraction governing the solution of the quantum Smoluchowski Eq. (13) to first order in $\Lambda$ only. Thus, substituting the Fourier expansion Eq. (23) into Eq. (13), we obtain the recurrence relation of the $C_{n}$ to first order in $\Lambda$ as

$$
\begin{aligned}
(i a+ & n) C_{n}-\frac{1}{2} i V_{0}\left[(1+n \Lambda) C_{n+1}+(1-n \Lambda) C_{n-1}\right]-i b_{1} V_{0}[(1 \\
& \left.+2 n \Lambda) C_{n+2}+(1-2 n \Lambda) C_{n-2}\right]-\frac{3}{2} i b_{2} V_{0}\left[(1+3 n \Lambda) C_{n+3}\right. \\
& \left.+(1-3 n \Lambda) C_{n-3}\right]=0 .
\end{aligned}
$$

The solution of this seven-term recurrence relation for the Fourier coefficients via matrix continued fractions is given in Appendix A. By orthogonality, using Eq. (15) the stationary average drift velocity of the particle $\langle\dot{x}\rangle_{0}$ is then given by the following linear combination of Fourier coefficients:

$$
\langle\dot{x}\rangle_{0}=a-V_{0} \operatorname{Re}\left[C_{1}+2 b_{1} C_{2}+3 b_{2} C_{3}\right] .
$$

\section{LINEAR AC RESPONSE OF THE QUANTUM BROWNIAN RATCHET}

Essentially, the same continued fraction as that resulting from Eq. (25) may be used to study quantum effects in the frequency dependence of the average drift velocity of the Brownian ratchet. Thus, we suppose that the ratchet is driven (in addition to the tilt $a$ ) by an unbiased ac force of amplitude $a^{\prime}$ and dimensionless angular frequency $\omega$. Hence, we require the time-dependent distribution $P(x, t)$. This may 
again be expanded as the complex Fourier series

$$
P(x, t)=\frac{1}{2 \pi} \sum_{n=-\infty}^{\infty} c_{n}(t) e^{i n x},
$$

where the Fourier coefficients which determine the characteristic function $c_{n}(t)$ of the displacement $x$ are

$$
c_{n}(t)=\int_{0}^{2 \pi} e^{-i n x} P(x, t) d x .
$$

Substituting Eq. (27) into Eq. (10), we find that the $c_{n}(t)$ satisfy the following ordinary differential-recurrence relation to first order in $\Lambda$

$$
\begin{aligned}
& \frac{d}{d t} c_{n}(t)+\left(i a n+n^{2}\right) c_{n}(t)-\frac{i n V_{0}}{2}\left[(1+n \Lambda) c_{n+1}(t)\right. \\
& \left.+(1-n \Lambda) c_{n-1}(t)\right]-i n b_{1} V_{0}\left[(1+2 n \Lambda) c_{n+2}(t)\right. \\
& \left.+(1-2 n \Lambda) c_{n-2}(t)\right]-\frac{3}{2} i n b_{2} V_{0}\left[(1+3 n \Lambda) c_{n+3}(t)\right. \\
& \left.\quad+(1-3 n \Lambda) c_{n-3}(t)\right]=0
\end{aligned}
$$

Equation (28) reduces to the previous time-independent recurrence relation (25) by simply omitting the time derivative. Moreover, it is essentially similar to the recurrence relation governing the quantum Brownian motion of a particle in a tilted cosine potential. The solution of that problem has recently been obtained in the context of quantum effects in the impedance of a Josephson junction [32,33] and long ago in the classical limit $[43,44]$.

We now specialize Eq. (28) (valid for arbitrary driving amplitude $a^{\prime}$ ) to small amplitudes such that $a^{\prime} \ll 1$. Hence the ratchet is only weakly perturbed, eliminating the phase modulation effects arising from the term $\operatorname{ianc}_{n}(t)$ in Eq. (28) which gives rise to an infinite number of harmonics in the ac response and frequency dependence of the dc response [45]. This procedure allows one to evaluate its linear response which may be extracted from Eq. (28) via perturbation theory in the oscillatory part $a^{\prime}$ of the tilt parameter as follows. We make the perturbation expansion (which implicitly assumes that all transients due to the imposition of the ac driving force have died away and that harmonics of that force are no longer generated)

$$
c_{n}(t)=C_{n}+a^{\prime} c_{n}(\omega) e^{i \omega t}+\cdots,
$$

where the stationary coefficients $C_{n}$ constitute the zeroth order of perturbation theory and satisfy the time-independent recurrence Eq. (25). Moreover, the particular Fourier coefficient $c_{0}(\omega)=0$, since $c_{0}(t)=1$ and $C_{0}=1$. Substituting Eq. (29) into the recurrence Eq. (28), with the replacement $a$ $\rightarrow a+a^{\prime} e^{i \omega t}$ and keeping only terms linear in the perturbation $a^{\prime}$, we have the forced recurrence relation for the Fourier amplitudes, namely,
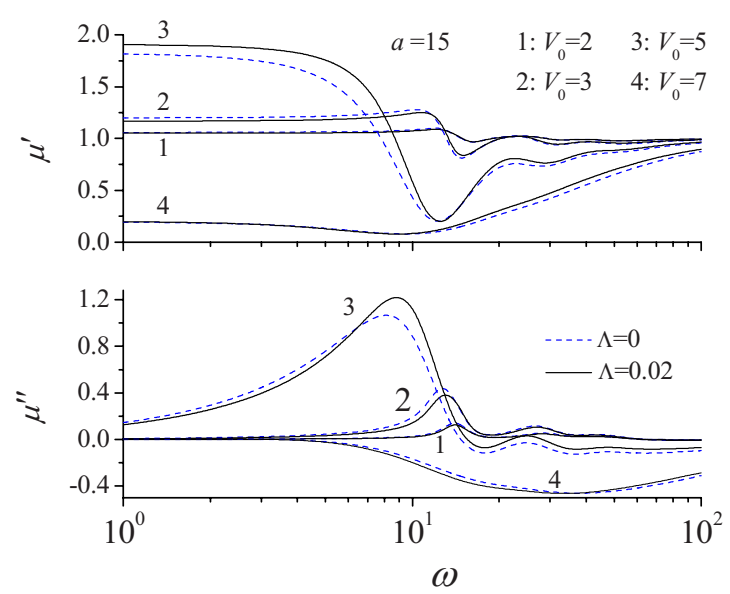

FIG. 3. (Color online) The real and imaginary parts of the linear dynamic mobility $\mu=\mu^{\prime}-i \mu^{\prime \prime}$ vs angular frequency $\omega$ in the ratchet potential $b_{1}=0.5$ and $b_{2}=0.5$ (see inset of Fig. 2) for various values of the barrier height $V_{0}=2$ (curves 1), $V_{0}=3$ (curves 2), $V_{0}=5$ (curves 3 ) and $V_{0}=7$ (curves 4 ), and the constant tilt $a=15$. The classical (dashed lines, $\Lambda=0$ ) and quantum (solid lines, $\Lambda$ $=0.02$ ) cases are shown.

$$
\begin{aligned}
(i a+ & n+i \omega / n) c_{n}(\omega)-\frac{1}{2} i V_{0}\left[(1+n \Lambda) c_{n+1}(\omega)\right. \\
& \left.+(1-n \Lambda) c_{n-1}(\omega)\right]-i b_{1} V_{0}\left[(1+2 n \Lambda) c_{n+2}(\omega)\right. \\
& \left.+(1-2 n \Lambda) c_{n-2}(\omega)\right]-\frac{3}{2} i b_{2} V_{0}\left[(1+3 n \Lambda) c_{n+3}(\omega)\right. \\
& \left.+(1-3 n \Lambda) c_{n-3}(\omega)\right]=-i C_{n} .
\end{aligned}
$$

In Appendix B, we describe the solution of the forced recurrence relation (30) via matrix continued fractions, noting that all the stationary solution coefficients $C_{n}$ are involved in the linear approximation. Recalling that the averaged dynamical equation in the presence of a weak ac force is

$$
\langle\dot{x}\rangle_{0}+\langle\dot{x}\rangle_{1}=a+a^{\prime} e^{i \omega t}-\left\langle\partial_{x} V\right\rangle_{0}-\left\langle\partial_{x} V\right\rangle_{1},
$$

where the zero subscript on the angular brackets denotes the average in the absence of the ac force, and the subscript 1 the portion of the average which is linear in $a^{\prime}$ we have

$$
\langle\dot{x}\rangle_{1}=\mu(\omega) a^{\prime} e^{i \omega t},
$$

where $\mu=\mu^{\prime}-i \mu^{\prime \prime}$ is the linear dynamic mobility given via orthogonality by the linear combination of Fourier amplitudes

$$
\begin{aligned}
\mu(\omega)= & 1-\frac{V_{0}}{2}\left\{c_{1}(\omega)+c_{1}^{*}(-\omega)+2 b_{1}\left[c_{2}(\omega)+c_{2}^{*}(-\omega)\right]\right. \\
& \left.+3 b_{2}\left[c_{3}(\omega)+c_{3}^{*}(-\omega)\right]\right\}
\end{aligned}
$$

where we have noted that $c_{n}(\omega)=c_{-n}^{*}(-\omega)$ and asterisk denotes complex conjugate.

We plot the real and imaginary parts of $\mu(\omega)$ given by Eq. (31) as a function of frequency for various values of the barrier height parameter $V_{0}$ in Fig. 3 and for various values of the tilt $a$ in Fig. 4. In Fig. 3 the curves show a pronounced minimum in $\mu^{\prime}(\omega)$ and maximum in $\mu^{\prime \prime}(\omega)$ accompanied by 

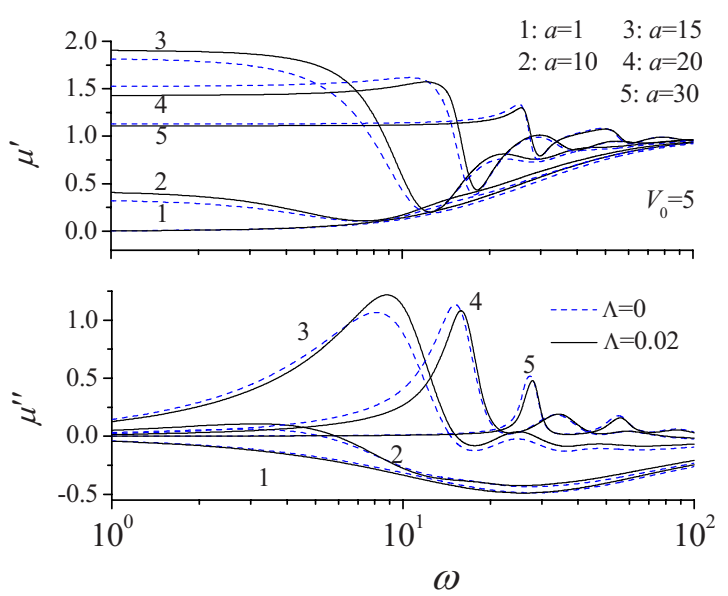

FIG. 4. (Color online) The real and imaginary parts of the linear dynamic mobility $\mu=\mu^{\prime}-i \mu^{\prime \prime}$ vs $\omega$ in the ratchet potential $V_{0}=5$, $b_{1}=0.5$ and $b_{2}=0.5$ for various values of the tilt $a=1$ (curves 1 ), $a=10$ (curves 2), $a=15$ (curves 3), $a=20$ (curves 4) and $a=30$ (curves 5). The classical (dashed lines, $\Lambda=0$ ) and quantum (solid lines, $\Lambda=0.02$ ) cases are shown.

subsidiary minima and maxima at higher frequencies. The quantum effects which increase the height of the maxima and shift them to higher frequencies (as in the related problem of the impedance of the Josephson junction) are most pronounced for moderate barrier heights $V_{0}=5$. They may be ascribed to dissipative tunneling at high temperatures near the top of a barrier created by the ratchet potential, effectively reducing the barrier height and representing a decrease in the damping factor. The quantum effects are smaller for the barriers created by the second and third harmonics of the potential and appear at higher frequencies. This behavior is replicated in Fig. 4 where the mobility is plotted for various values of the tilt. Again, as is the experience with the Josephson junction, quantum effects are most prominent at moderate tilts, causing the principal maximum in the imaginary part to shift to higher frequencies with increasing tilt.

\section{NONLINEAR RESPONSE OF THE QUANTUM BROWNIAN RATCHET}

In the previous section we considered the quantum Brownian ratchet with arbitrary tilt perturbed by a weak alternating force $a^{\prime} e^{i \omega t}$ so that $a^{\prime} \ll 1$. Hence the response is linear in the first order of perturbation in $a^{\prime}$. In order to calculate the frequency response of the ratchet for arbitrary driving amplitude $a^{\prime}$ we must consider the nonlinear solution. Since we are again concerned with the stationary response, where the driving force has been applied for a very long time, we may ignore transient effects and seek a solution independent of the initial conditions. Thus, we make the temporal Fourier expansion

$$
c_{n}(t)=\sum_{k=-\infty}^{\infty} c_{n}^{k}(\omega) e^{i k \omega t},
$$

where the infinite sum in $k$ accounts for the infinite number of harmonics of the stimulus produced by the nonlinear char- acteristics of the Brownian ratchet and the Fourier coefficients have the property $c_{n}^{k}(\omega)=c_{-n}^{k *}(-\omega)=c_{-n}^{-k *}(\omega)$, because $P(x, t)$ must be real. The harmonic generating property of the nonlinear response is particularly obvious if one simply regards Eq. (28) as a differential equation for a linear timevarying system forced by the recurring terms. The integrating factor thus involves a time-varying double transcendental function, which causes phase modulation of the successive approximations solution. This has been illustrated in detail in Ref. [46] in connection with the classical treatment of the Josephson junction in the zero capacitance limit. We remark that frequency dependence of the dc term is more or less a universal phenomenon in nonlinear systems driven by alternating forces, occurring in systems as diverse as the Josephson junction, ring-laser gyros, superparamagnetic nanoparticles [7], and the Kerr-effect response of dipoles in a mean field potential [47]. In ratchets, the frequency dependence is due to the ever prevailing modulation of the static characteristics resulting in loss of phase locking, e.g., the well known Shapiro steps in the frequency-dependent dc current-voltage characteristic of the Josephson junction [48]. However, the frequency-dependent dc response of electric dipoles is inherently simpler than that of the ratchet because the integrating factor in the sense mentioned above is essentially time invariant. The effect of the nonlinearity in this case is to give rise to amplitude rather than phase modulation [45].

By substituting Eq. (32) into Eq. (28) and letting the tilt $a \rightarrow a+a^{\prime} \cos \omega t$, we have the dual-index recurrence relation for the Fourier amplitudes $c_{n}^{k}(\omega)$, viz.,

$$
\begin{aligned}
(i a+ & n+i k \omega / n) c_{n}^{k}(\omega)+\frac{1}{2} i a^{\prime}\left[c_{n}^{k+1}(\omega)+c_{n}^{k-1}(\omega)\right] \\
& -\frac{1}{2} i V_{0}\left[(1+n \Lambda) c_{n+1}^{k}(\omega)+(1-n \Lambda) c_{n-1}^{k}(\omega)\right] \\
& -i b_{1} V_{0}\left[(1+2 n \Lambda) c_{n+2}^{k}(\omega)+(1-2 n \Lambda) c_{n-2}^{k}(\omega)\right] \\
& -\frac{3}{2} i b_{2} V_{0}\left[(1+3 n \Lambda) c_{n+3}^{k}(\omega)+(1-3 n \Lambda) c_{n-3}^{k}(\omega)\right]=0,
\end{aligned}
$$

where $n$ and $k$ are integers varying from $-\infty$ to $\infty, c_{0}^{0}(\omega)=1$, and $c_{0}^{k}(\omega)=0(k>0)$. We present the recurrence relation (33) above in matrix continued fraction form in Appendix C, noting that supermatrices will now be involved as a result of the dual-indices $n$ and $k$. Finally, from Eq. (15) (replacing $a$ with $\left.a+a^{\prime} \cos \omega t\right)$ the average drift velocity $\langle\dot{x}\rangle$ in the presence of an alternating force $a^{\prime} \cos \omega t$ is given by

$$
\langle\dot{x}\rangle=a+a^{\prime} \cos \omega t-\frac{1}{2 \pi} \int_{0}^{2 \pi} V^{\prime}(x) \sum_{n=-\infty}^{\infty} \sum_{k=-\infty}^{\infty} c_{n}^{k}(\omega) e^{i k \omega t} e^{i n x} d x
$$

Substituting the explicit form of the potential, Eq. (11), and using orthogonality we have 


$$
\begin{aligned}
\langle\dot{x}\rangle= & a+a^{\prime} \cos \omega t-\frac{V_{0}}{2} \sum_{k=-\infty}^{\infty} e^{i k \omega t}\left[c_{1}^{k}(\omega)+c_{-1}^{k}(\omega)+2 b_{1} c_{2}^{k}(\omega)\right. \\
& \left.+2 b_{1} c_{-2}^{k}(\omega)+3 b_{2} c_{3}^{k}(\omega)+3 b_{2} c_{-3}^{k}(\omega)\right],
\end{aligned}
$$

which may be expanded as

$$
\begin{aligned}
\langle\dot{x}\rangle= & a-V_{0} \operatorname{Re}\left[c_{1}^{0}(\omega)+2 b_{1} c_{2}^{0}(\omega)+3 b_{2} c_{3}^{0}(\omega)\right] \\
& +\left\{1-\frac{V_{0}}{a^{\prime}}\left[c_{1}^{1}(\omega)+c_{-1}^{1}(\omega)+2 b_{1} c_{2}^{1}(\omega)+2 b_{1} c_{-2}^{1}(\omega)\right.\right. \\
& \left.\left.+3 b_{2} c_{3}^{1}(\omega)+3 b_{2} c_{-3}^{1}(\omega)\right]\right\} \frac{a^{\prime}}{2} e^{i \omega t}+\left\{1-\frac{V_{0}}{a^{\prime}}\left[c_{1}^{-1}(\omega)\right.\right. \\
& +c_{-1}^{-1}(\omega)+2 b_{1} c_{2}^{-1}(\omega)+2 b_{1} c_{-2}^{-1}(\omega)+3 b_{2} c_{3}^{-1}(\omega) \\
& \left.\left.+3 b_{2} c_{-3}^{-1}(\omega)\right]\right\} \frac{a^{\prime}}{2} e^{-i \omega t}+\frac{V_{0}}{2} \sum_{k=2}^{\infty}\left\{e ^ { i k \omega t } \left[c_{1}^{k}(\omega)+c_{-1}^{k}(\omega)\right.\right. \\
& \left.+2 b_{1} c_{2}^{k}(\omega)+2 b_{1} c_{-2}^{k}(\omega)+3 b_{2} c_{3}^{k}(\omega)+3 b_{2} c_{-3}^{k}(\omega)\right] \\
& +e^{-i k \omega t}\left[c_{1}^{-k}(\omega)+c_{-1}^{-k}(\omega)+2 b_{1} c_{2}^{-k}(\omega)+2 b_{1} c_{-2}^{-k}(\omega)\right. \\
& \left.\left.+3 b_{2} c_{3}^{-k}(\omega)+3 b_{2} c_{-3}^{-k}(\omega)\right]\right\} .
\end{aligned}
$$

The first line of Eq. (34) represents the frequency-dependent dc term $\langle\dot{x}\rangle_{d c}$, corresponding to the stationary frequencyindependent response Eq. (26). The next two terms represent the response at the fundamental frequency and although ostensibly of the same form as the linear dynamic mobility Eq. (31), differ in principle from it because each Fourier component now contains contributions from all other harmonics (which arise on account of the nonlinear behavior, see Ref. [45]). The remaining summation represents the infinite number of harmonics of the driving signal, generated by nonlinearity.

We illustrate the frequency dependence of the dc term $\langle\dot{x}\rangle_{d c}$ for various values of the driving amplitude $a^{\prime}$, plotted as a function of the tilt $a$ in Fig. 5 and as a function of the frequency $\omega$ in Fig. 6. In Fig. 5, the well known Shapiro steps (curves 1-4) familiar in the dc current/voltage characteristics of a Josephson junction for strong ac driving are illustrated. The steps appear as a result of phase modulation due to nonlinear effects and represent loss of phase locking (phase slips) at harmonics of the ac driving force. This result is in marked contrast to the frequency-independent average dc drift velocity Eq. (26) (curve 0) where the steps do not exist. The quantum effects are most pronounced as with our previous experience $[32,33]$ of the Josephson junction for relatively small values of the driving force (although strong enough to cause nonlinear behavior) becoming almost imperceptible for very large driving forces, where they tend to be masked by the nonlinearity. In Fig. 6, we show the frequency response of the ac-induced average dc mobility. The quantum effects again tend to enhance the ac-induced dc mobility in comparison with the classical case. They are most pronounced at frequencies corresponding to the ac-induced peaks in the dc response, increasing the effective peak mobility for moderate driving due to the reduction in damping, arising from high temperature dissipative tunneling near the top of a barrier. The quantum effects also cause slower fal-

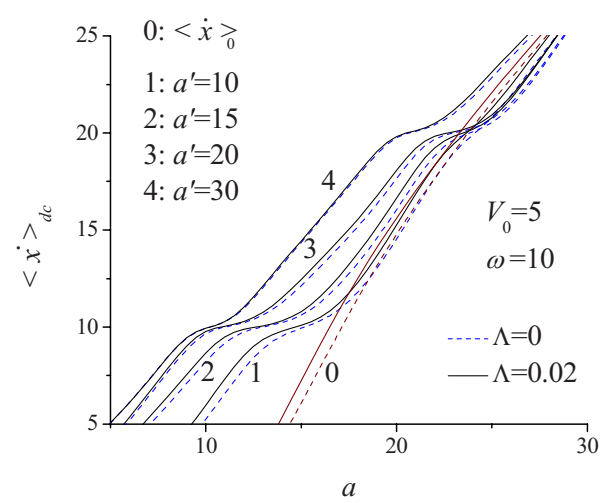

FIG. 5. (Color online) The frequency-dependent dc term of the average drift velocity $\langle\dot{x}\rangle_{d c}$ vs the tilt $a$ in the ratchet potential $V_{0}$ $=5, b_{1}=0.5$, and $b_{2}=0.5$ for various values of the ac driving amplitude $a^{\prime}=10$ (curves 1), $a^{\prime}=15$ (curves 2), $a^{\prime}=20$ (curves 3 ) and $a^{\prime}=30$ (curves 4) for fixed frequency $\omega=10$. The Shapiro steps (curves 1-4) appear as a result of phase modulation due to nonlinear effects, in contrast to the frequency-independent average drift velocity $\langle\dot{x}\rangle_{0}$ (curve 0) plotted using Eq. (26). The classical (dashed lines, $\Lambda=0$ ) and quantum (solid lines, $\Lambda=0.02$ ) cases are shown.

loffs of the dc response and again become very small as the amplitude of the driving force is increased due to the masking effects of the nonlinearity (compare curves 1 and 5 of Fig. 6). The behavior at the peak frequencies seems to be entirely consistent with the effective reduction in damping due to tunneling mentioned above resulting in increased mobility so enhancing the peaks just as in the Josephson junction. The same behavior is evident in the imaginary part of the nonlinear mobility at the fundamental frequency of the driving force, where once again the maxima in the spectra are enhanced with the quantum effects being most pronounced for moderate driving as shown in Fig. 7.

\section{CONCLUSIONS}

We have demonstrated how the quantum Smoluchowski equation may be used to calculate the average drift velocity of a quantum ratchet for wide ranges of the barrier height, tilt

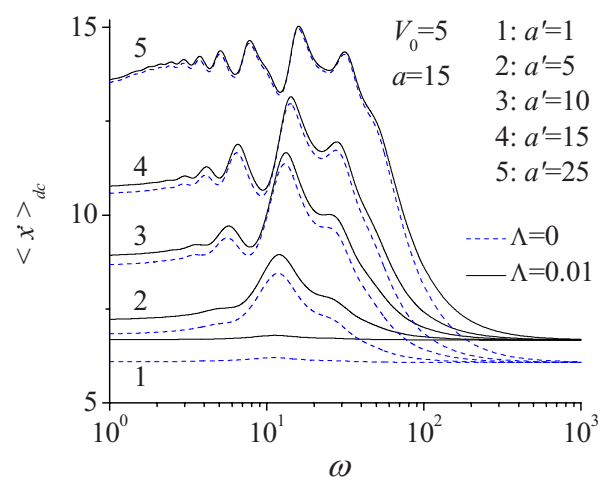

FIG. 6. (Color online) The frequency-dependent dc term of the average drift velocity $\langle\dot{x}\rangle_{d c}$ vs angular frequency $\omega$ in the ratchet potential $V_{0}=5, b_{1}=0.5$, and $b_{2}=0.5$ for various values of the ac driving amplitude $a^{\prime}=1$ (curves 1), $a^{\prime}=5$ (curves 2), $a^{\prime}=10$ (curves 3), $a^{\prime}=15$ (curves 4), and $a^{\prime}=25$ (curves 5), and the constant tilt $a=15$. The classical (dashed lines, $\Lambda=0$ ) and quantum (solid lines, $\Lambda=0.01)$ cases are shown. 

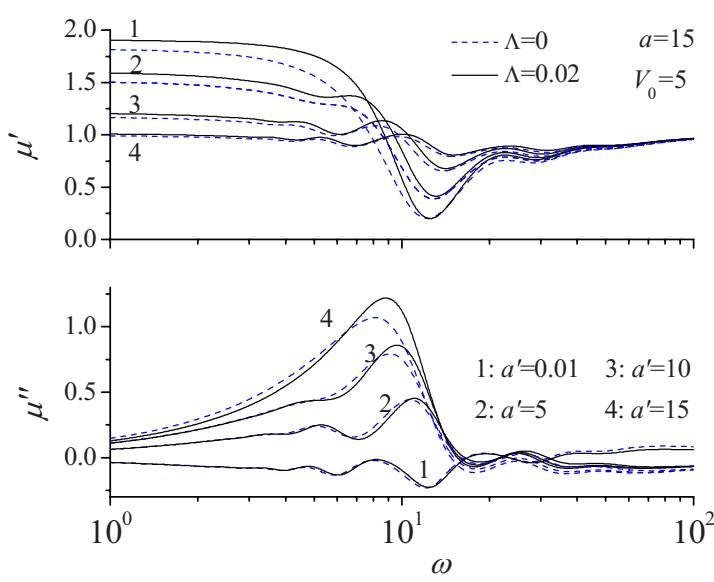

FIG. 7. (Color online) The real and imaginary parts of the nonlinear dynamic mobility $\mu=\mu^{\prime}-i \mu^{\prime \prime}$ vs angular frequency $\omega$ in the ratchet potential, $V_{0}=5 b_{1}=0.5$ and $b_{2}=0.5$ for various values of the stimulus amplitude $a^{\prime}=0.01$ (curves 1 ), $a^{\prime}=5$ (curves 2), $a^{\prime}=10$ (curves 3 ) and $a^{\prime}=15$ (curves 4 ), and the constant tilt $a=15$. The classical (dashed lines, $\Lambda=0$ ) and quantum (solid lines, $\Lambda=0.02$ ) cases are shown. In the limit of $a^{\prime} \ll 1$ the linear response is reproduced, i.e., curves 1 correspond exactly to curves 3 of Fig. 3.

and ac driving parameters. It appears that quantum effects reduce the average drift velocity in the slow-switching limit of a two-state driving force in comparison to the classical case. In general, as far as the frequency response is concerned, the quantum effects modify the spectra of the mobility. The origin of this phenomenon appears to be due a reduction of the barrier height, representing a decrease in the damping factor and thus an increase in the dynamic mobility (due to high temperature tunneling near the top of the barrier) over the corresponding classical results for a given bias. Furthermore, in accordance with previous experience [32,33] concerning the ac response of the Josephson junction, the quantum effects are most pronounced for moderate ac driving forces, generally diminishing with increasing ac amplitude as they now tend to be masked by the extreme nonlinear behavior. A most interesting additional feature of the nonlinear frequency-dependent dc response to a strong ac driving force in both the quantum and classical cases is the appearance of Shapiro-like steps $[46,48]$ (due to loss of phase locking at harmonics of the driving force) in the average dc drift velocity versus tilt characteristic. This phenomenon is exactly analogous to the Shapiro steps [48] in the dc current/ voltage characteristics of the Josephson junction for strong ac driving and should be amenable to experimental verification as a universal feature of the dc response associated with strong ac driving of particles in tilted periodic potentials.

\section{ACKNOWLEDGMENTS}

The Ireland-France IRCSET/EGIDE scheme and the Royal Irish Academy (mobility grant 2009) are thanked for their financial support for this work. L.C. acknowledges IRCSET for support.

\section{APPENDIX A: STATIONARY SOLUTION-MATRIX CONTINUED FRACTION SOLUTION OF EQ. (25)}

The recurrence relation for the $C_{n}$ may be represented as the seven-term recurrence relation

$$
\begin{aligned}
q_{n} C_{n} & +q_{n}^{+} C_{n+1}+q_{n}^{-} C_{n-1}+2 b_{1} q_{2 n}^{+} C_{n+2}+2 b_{1} q_{2 n}^{-} C_{n-2} \\
& +3 b_{2} q_{3 n}^{+} C_{n+3}+3 b_{2} q_{3 n}^{-} C_{n-3}=0
\end{aligned}
$$

where $q_{n}=i a+n$ and $q_{n}^{ \pm}=-i V_{0}(1 \pm n \Lambda) / 2$. By introducing the column vectors $\mathbf{C}_{n}$ (which are in general complex)

$$
\mathbf{C}_{ \pm n}=\left(\begin{array}{c}
C_{ \pm 3 n} \\
C_{ \pm(3 n-1)} \\
C_{ \pm(3 n-2)}
\end{array}\right), \quad n>1,
$$

with $\mathbf{C}_{0}=(1)$ and the property $\mathbf{C}_{-n}=\mathbf{C}_{n}^{*}$ Eq. (A1) may be rewritten in terms of the inhomogeneous and homogeneous matrix three-term recurrence relations

$$
\begin{aligned}
& \mathbf{Q}_{1}^{-} \mathbf{C}_{0}+\mathbf{Q}_{1} \mathbf{C}_{1}+\mathbf{Q}_{1}^{+} \mathbf{C}_{2}=-\mathbf{F} \mathbf{C}_{1}^{*} \quad(n=1), \\
& \mathbf{Q}_{n}^{-} \mathbf{C}_{n-1}+\mathbf{Q}_{n} \mathbf{C}_{n}+\mathbf{Q}_{n}^{+} \mathbf{C}_{n+1}=0 \quad(n>1) .
\end{aligned}
$$

where the matrices $\mathbf{Q}_{n}$ and $\mathbf{Q}_{n}^{ \pm}$are given by

$$
\begin{gathered}
\mathbf{Q}_{n}=\left(\begin{array}{ccc}
q_{3 n} & q_{3 n}^{-} & 2 b_{1} q_{2(3 n)}^{-} \\
q_{3 n-1}^{+} & q_{3 n-1} & q_{3 n-1}^{-} \\
2 b_{1} q_{2(3 n-2)}^{+} & q_{3 n-2}^{+} & q_{3 n-2}
\end{array}\right), \\
\mathbf{Q}_{n}^{+}=\left(\begin{array}{ccc}
3 b_{2} q_{3(3 n)}^{+} & 2 b_{1} q_{2(3 n)}^{+} & q_{3 n}^{+} \\
0 & 3 b_{2} q_{3(3 n-1)}^{+} & 2 b_{1} q_{2(3 n-1)}^{+} \\
0 & 0 & 3 b_{2} q_{3(3 n-2)}^{+}
\end{array}\right), \\
\mathbf{Q}_{n}^{-}=\left(\begin{array}{ccc}
3 b_{2} q_{3(3 n)}^{-} & 0 & 0 \\
2 b_{1} q_{2(3 n-1)}^{-} & 3 b_{2} q_{3(3 n-1)}^{-} & 0 \\
q_{3 n-2}^{-} & 2 b_{1} q_{2(3 n-2)}^{-} & 3 b_{2} q_{3(3 n-2)}^{-}
\end{array}\right),
\end{gathered}
$$

with $\mathbf{Q}_{1}^{-}$and $\mathbf{F}$ defined as

$$
\mathbf{Q}_{1}^{-}=\left(\begin{array}{c}
3 b_{2} q_{9}^{-} \\
2 b_{1} q_{4}^{-} \\
q_{1}^{-}
\end{array}\right), \quad \mathbf{F}=\left(\begin{array}{ccc}
0 & 0 & 0 \\
0 & 0 & 3 b_{2} q_{6}^{-} \\
0 & 3 b_{2} q_{3}^{-} & 2 b_{1} q_{2}^{-}
\end{array}\right)
$$

Introducing matrices $\mathbf{S}_{n}=\mathbf{C}_{n}\left(\mathbf{C}_{n-1}\right)^{-1}$ and $\boldsymbol{\Delta}_{n}=\mathbf{S}_{n}\left(\mathbf{Q}_{n}^{-}\right)^{-1}$ we then have the solution of the homogeneous Eq. (A3) for $\mathbf{C}_{n}(n>1)$ in terms of the matrix continued fractions 


$$
\mathbf{C}_{n}=\boldsymbol{\Delta}_{n} \mathbf{Q}_{n}^{-} \boldsymbol{\Delta}_{n-1} \mathbf{Q}_{n-1}^{-} \ldots \boldsymbol{\Delta}_{2} \mathbf{Q}_{2}^{-} \mathbf{C}_{1},
$$

where $\Delta_{n}$ is the infinite matrix continued fraction defined by the recurrence equation

$$
\boldsymbol{\Delta}_{n}=\left(-\mathbf{Q}_{n}-\mathbf{Q}_{n}^{+} \boldsymbol{\Delta}_{n+1} \mathbf{Q}_{n+1}^{-}\right)^{-1} .
$$

The inhomogeneous Eq. (A2) for the vector $\mathbf{C}_{1}$ then has solution

$$
\mathbf{C}_{1}=\boldsymbol{\Delta}_{1}\left(\mathbf{Q}_{1}^{-} \mathbf{C}_{0}+\mathbf{F} \mathbf{C}_{1}^{*}\right) \text {. }
$$

Next, we eliminate by representing the various complex vectors and matrices in terms of their real and imaginary parts $\mathbf{C}_{1}=\mathbf{C}_{1}^{\prime}+i \mathbf{C}_{1}^{\prime \prime}, \boldsymbol{\Delta}_{1} \mathbf{Q}_{1}^{-}=\mathbf{S}_{1}^{\prime}+i \mathbf{S}_{1}^{\prime \prime}$, and $\boldsymbol{\Delta}_{1} \mathbf{F}=\mathbf{F}^{\prime}+i \mathbf{F}^{\prime \prime}$ so obtaining from Eq. (A6) simultaneous equations for the unknowns $\mathbf{C}_{1}^{\prime}$ and $\mathbf{C}_{1}^{\prime \prime}$ comprising the desired column vector $\mathbf{C}_{1}$, namely

$$
\begin{aligned}
& \left(\mathbf{I}-\mathbf{F}^{\prime}\right) \mathbf{C}_{1}^{\prime}-\mathbf{F}^{\prime \prime} \mathbf{C}_{1}^{\prime \prime}=\mathbf{S}_{1}^{\prime} \mathbf{C}_{0}, \\
& \left(\mathbf{I}+\mathbf{F}^{\prime}\right) \mathbf{C}_{1}^{\prime \prime}-\mathbf{F}^{\prime \prime} \mathbf{C}_{1}^{\prime}=\mathbf{S}_{1}^{\prime \prime} \mathbf{C}_{0},
\end{aligned}
$$

where $\mathbf{I}$ is the unit matrix. Solving Eqs. (A7) and (A8) for $\mathbf{C}_{1}^{\prime}$ and $\mathbf{C}_{1}^{\prime \prime}$ we have in terms of known matrices

$$
\begin{aligned}
& \mathbf{C}_{1}^{\prime}=\left[\mathbf{I}-\mathbf{F}^{\prime}-\mathbf{F}^{\prime \prime}\left(\mathbf{I}+\mathbf{F}^{\prime}\right)^{-1} \mathbf{F}^{\prime \prime}\right]^{-1}\left[\mathbf{S}_{1}^{\prime}+\mathbf{F}^{\prime \prime}\left(\mathbf{I}+\mathbf{F}^{\prime}\right)^{-1} \mathbf{S}_{1}^{\prime \prime}\right] \mathbf{C}_{0}, \\
& \mathbf{C}_{1}^{\prime \prime}=\left[\mathbf{I}+\mathbf{F}^{\prime}-\mathbf{F}^{\prime \prime}\left(\mathbf{I}-\mathbf{F}^{\prime}\right)^{-1} \mathbf{F}^{\prime \prime}\right]^{-1}\left[\mathbf{S}_{1}^{\prime \prime}+\mathbf{F}^{\prime \prime}\left(\mathbf{I}-\mathbf{F}^{\prime}\right)^{-1} \mathbf{S}_{1}^{\prime}\right] \mathbf{C}_{0} .
\end{aligned}
$$

APPENDIX B: LINEAR RESPONSE-MATRIX CONTINUED FRACTION SOLUTION OF EQ. (30)

In like manner, the eight-term recurrence relation for the Fourier amplitudes $c_{n}(\omega)$, Eq. (30) can be rearranged as

$$
\begin{aligned}
& z_{n}(\omega) c_{n}(\omega)+q_{n}^{+} c_{n+1}(\omega)+q_{n}^{-} c_{n-1}(\omega)+2 b_{1}\left[q_{2 n}^{+} c_{n+2}(\omega)\right. \\
& \left.\quad+q_{2 n}^{-} c_{n-2}(\omega)\right]+3 b_{2}\left[q_{3 n}^{+} c_{n+3}(\omega)+q_{3 n}^{-} c_{n-3}(\omega)\right]=-i C_{n}
\end{aligned}
$$

where $z_{n}(\omega)=i a+n+i \omega / n$ and $q_{n}^{ \pm}=-i V_{0}(1 \pm n \Lambda) / 2$. By introducing column vectors $\mathbf{C}_{n}(\omega)$ and the known stationary (or zero order of perturbation solution) column vector $\mathbf{C}_{n}^{0}$ [as calculated in Eqs. (A4) and (A6)]

$$
\begin{gathered}
\mathbf{C}_{ \pm n}(\omega)=\left(\begin{array}{c}
c_{ \pm 3 n}(-\omega) \\
c_{ \pm 3 n}(\omega) \\
c_{ \pm(3 n-1)}(-\omega) \\
c_{ \pm(3 n-1)}(\omega) \\
c_{ \pm(3 n-2)}(-\omega) \\
c_{ \pm(3 n-2)}(\omega)
\end{array}\right), \quad \mathbf{C}_{ \pm n}^{0}=i\left(\begin{array}{c}
C_{ \pm 3 n} \\
C_{ \pm 3 n} \\
C_{ \pm(3 n-1)} \\
C_{ \pm(3 n-1)} \\
C_{ \pm(3 n-2)} \\
C_{ \pm(3 n-2)}
\end{array}\right), \\
n>1,
\end{gathered}
$$

with $\mathbf{C}_{0}=(0)$ and the property $\mathbf{C}_{-n}(\omega)=\mathbf{C}_{n}^{*}(-\omega)$, Eq. (B1) may be written as the inhomogeneous matrix three-term recurrence relations

$$
\begin{gathered}
\mathbf{Q}_{1} \mathbf{C}_{1}+\mathbf{Q}_{1}^{+} \mathbf{C}_{2}=-\left(\mathbf{C}_{1}^{0}+\mathbf{F} \mathbf{C}_{1}^{*}\right) \quad(n=1), \\
\mathbf{Q}_{n}^{-} \mathbf{C}_{n-1}+\mathbf{Q}_{n} \mathbf{C}_{n}+\mathbf{Q}_{n}^{+} \mathbf{C}_{n+1}=-\mathbf{C}_{n}^{0} \quad(n>1) .
\end{gathered}
$$

The matrices $\mathbf{Q}_{n}$ and $\mathbf{Q}_{n}^{ \pm}$are given by

$$
\begin{gathered}
\mathbf{Q}_{n}=\left(\begin{array}{cccccc}
z_{3 n}(-\omega) & 0 & q_{3 n}^{-} & 0 & 2 b_{1} q_{2(3 n)}^{-} & 0 \\
0 & z_{3 n}(\omega) & 0 & q_{3 n}^{-} & 0 & 2 b_{1} q_{2(3 n)}^{-} \\
q_{3 n-1}^{+} & 0 & z_{3 n-1}(-\omega) & 0 & q_{3 n-1}^{-} & 0 \\
0 & q_{3 n-1}^{+} & 0 & z_{3 n-1}(\omega) & 0 & q_{3 n-1}^{-} \\
2 b_{1} q_{2(3 n-2)}^{+} & 0 & q_{3 n-2}^{+} & 0 & z_{3 n-2}(-\omega) & 0 \\
0 & 2 b_{1} q_{2(3 n-2)}^{+} & 0 & q_{3 n-2}^{+} & 0 & z_{3 n-2}(\omega)
\end{array}\right), \\
\mathbf{Q}_{n}^{+}=\left(\begin{array}{cccccc}
3 b_{2} q_{3(3 n)}^{+} & 0 & 2 b_{1} q_{2(3 n)}^{+} & 0 & q_{3 n}^{+} & 0 \\
0 & 3 b_{2} q_{3(3 n)}^{+} & 0 & 2 b_{1} q_{2(3 n)}^{+} & 0 & q_{3 n}^{+} \\
0 & 0 & 3 b_{2} q_{3(3 n-1)}^{+} & 0 & 2 b_{1} q_{2(3 n-1)}^{+} & 0 \\
0 & 0 & 0 & 3 b_{2} q_{3(3 n-1)}^{+} & 0 & 2 b_{1} q_{2(3 n-1)}^{+} \\
0 & 0 & 0 & 0 & 3 b_{2} q_{3(3 n-2)}^{+} & 0 \\
0 & 0 & 0 & 0 & 0 & 3 b_{2} q_{3(3 n-2)}^{+}
\end{array}\right),
\end{gathered}
$$




$$
\mathbf{Q}_{n}^{-}=\left(\begin{array}{cccccc}
3 b_{2} q_{3(3 n)}^{-} & 0 & 0 & 0 & 0 & 0 \\
0 & 3 b_{2} q_{3(3 n)}^{-} & 0 & 0 & 0 & 0 \\
2 b_{1} q_{2(3 n-1)}^{-} & 0 & 3 b_{2} q_{3(3 n-1)}^{-} & 0 & 0 & 0 \\
0 & 2 b_{1} q_{2(3 n-1)}^{-} & 0 & 3 b_{2} q_{3(3 n-1)}^{-} & 0 & 0 \\
q_{3 n-2}^{-} & 0 & 2 b_{1} q_{2(3 n-2)}^{-} & 0 & 3 b_{2} q_{3(3 n-2)}^{-} & 0 \\
0 & q_{3 n-2}^{-} & 0 & 2 b_{1} q_{2(3 n-2)}^{-} & 0 & 3 b_{2} q_{3(3 n-2)}^{-}
\end{array}\right),
$$

with $\mathbf{F}$ defined as

$$
\mathbf{F}=\left(\begin{array}{cccccc}
0 & 0 & 0 & 0 & 0 & 0 \\
0 & 0 & 0 & 0 & 0 & 0 \\
0 & 0 & 0 & 0 & 0 & 3 b_{2} q_{6}^{-} \\
0 & 0 & 0 & 0 & 3 b_{2} q_{6}^{-} & 0 \\
0 & 0 & 0 & 3 b_{2} q_{3}^{-} & 0 & 2 b_{1} q_{2}^{-} \\
0 & 0 & 3 b_{2} q_{3}^{-} & 0 & 2 b_{1} q_{2}^{-} & 0
\end{array}\right) .
$$

where $z_{n}^{k}(\omega)=i a+n+i k \omega / n$ and $q_{n}^{ \pm}=-i V_{0}(1 \pm n \Lambda) / 2$. We may rewrite the above nine-term recurrence relation as a seven-term matrix recurrence relation

$$
\begin{aligned}
\mathbf{q}_{n} \mathbf{c}_{n}(\omega)+\mathbf{q}_{n}^{+} \mathbf{c}_{n+1}(\omega)+\mathbf{q}_{n}^{-} \mathbf{c}_{n-1}(\omega)+2 b_{1}\left[\mathbf{q}_{2 n}^{+} \mathbf{c}_{n+2}(\omega)\right. \\
\left.+\mathbf{q}_{2 n}^{-} \mathbf{c}_{n-2}(\omega)\right]+3 b_{2}\left[\mathbf{q}_{3 n}^{+} \mathbf{c}_{n+3}(\omega)+\mathbf{q}_{3 n}^{-} \mathbf{c}_{n-3}(\omega)\right]=0,
\end{aligned}
$$

where

$$
\mathbf{c}_{n}(\omega)=\left(\begin{array}{c}
\vdots \\
c_{n}^{-1}(\omega) \\
c_{n}^{0}(\omega) \\
c_{n}^{1}(\omega) \\
\vdots
\end{array}\right), \quad \mathbf{c}_{0}=\left(\begin{array}{c}
\vdots \\
0 \\
1 \\
0 \\
\vdots
\end{array}\right),
$$

Noting that $\mathbf{C}_{0}=(0)$, Eq. (B2) can be solved for $\mathbf{C}_{1}$ yielding

$$
\mathbf{C}_{1}=\boldsymbol{\Delta}_{1}\left(\mathbf{C}_{1}^{0}+\mathbf{X}_{1}+\mathbf{F C}_{1}^{*}\right),
$$

where

$$
\mathbf{X}_{1}=\sum_{k=1}^{\infty}\left(\prod_{p=1}^{k} \mathbf{Q}_{p}^{+} \boldsymbol{\Delta}_{p+1}\right) \mathbf{C}_{k+1}^{0} .
$$

Representing the complex vectors and matrices in Eq. (B5) as $\mathbf{C}_{1}=\mathbf{C}_{1}^{\prime}+i \mathbf{C}_{1}^{\prime \prime}, \boldsymbol{\Delta}_{1}\left(\mathbf{C}_{1}^{0}+\mathbf{X}_{1}\right)=\mathbf{C}_{1}^{0^{\prime}}+i \mathbf{C}_{1}^{0^{\prime \prime}}$, and $\boldsymbol{\Delta}_{1} \mathbf{F}=\mathbf{F}^{\prime}+i \mathbf{F}^{\prime \prime}$, we can solve for $\mathbf{C}_{1}^{\prime}$ and $\mathbf{C}_{1}^{\prime \prime}$ by equating coefficients to find in terms of known matrices

$$
\begin{aligned}
& \mathbf{C}_{1}^{\prime}=\left[\mathbf{I}-\mathbf{F}^{\prime}-\mathbf{F}^{\prime \prime}\left(\mathbf{I}+\mathbf{F}^{\prime}\right)^{-1} \mathbf{F}^{\prime \prime}\right]^{-1}\left[\mathbf{C}_{1}^{0^{\prime}}+\mathbf{F}^{\prime \prime}\left(\mathbf{I}+\mathbf{F}^{\prime}\right)^{-1} \mathbf{C}_{1}^{0^{\prime \prime}}\right], \\
& \mathbf{C}_{1}^{\prime \prime}=\left[\mathbf{I}+\mathbf{F}^{\prime}-\mathbf{F}^{\prime \prime}\left(\mathbf{I}-\mathbf{F}^{\prime}\right)^{-1} \mathbf{F}^{\prime \prime}\right]^{-1}\left[\mathbf{C}_{1}^{0^{\prime \prime}}+\mathbf{F}^{\prime \prime}\left(\mathbf{I}-\mathbf{F}^{\prime}\right)^{-1} \mathbf{C}_{1}^{0^{\prime}}\right] .
\end{aligned}
$$

\section{APPENDIX C: NONLINEAR RESPONSE-MATRIX CONTINUED FRACTION SOLUTION OF EQ. (33)}

As before, we represent the dual-index recurrence relation for the Fourier amplitudes as

$$
\begin{aligned}
& z_{n}^{k}(\omega) c_{n}^{k}(\omega)+\frac{i a^{\prime}}{2}\left[c_{n}^{k+1}(\omega)+c_{n}^{k-1}(\omega)\right]+q_{n}^{+} c_{n+1}^{k}(\omega)+q_{n}^{-} c_{n-1}^{k}(\omega) \\
& \quad+2 b_{1}\left[q_{2 n}^{+} c_{n+2}^{k}(\omega)+q_{2 n}^{-} c_{n-2}^{k}(\omega)\right]+3 b_{2}\left[q_{3 n}^{+} c_{n+3}^{k}(\omega)\right. \\
& \left.\quad+q_{3 n}^{-} c_{n-3}^{k}(\omega)\right]=0,
\end{aligned}
$$

$$
\begin{gathered}
\mathbf{q}_{n}=\left(\begin{array}{ccccc}
\ddots & \vdots & \vdots & \vdots & . \\
\cdots & z_{n}^{-1}(\omega) & i a^{\prime} / 2 & 0 & \cdots \\
\cdots & i a^{\prime} / 2 & z_{n}^{0}(\omega) & i a^{\prime} / 2 & \cdots \\
\cdots & 0 & i a^{\prime} / 2 & z_{n}^{1}(\omega) & \cdots \\
. & \vdots & \vdots & \vdots & \ddots
\end{array}\right), \\
\mathbf{q}_{n}^{ \pm}=\left(\begin{array}{ccccc}
\ddots & \vdots & \vdots & \vdots & . \\
\cdots & q_{n}^{ \pm} & 0 & 0 & \cdots \\
\cdots & 0 & q_{n}^{ \pm} & 0 & \cdots \\
\cdots & 0 & 0 & q_{n}^{ \pm} & \cdots \\
. & \vdots & \vdots & \vdots & \ddots
\end{array}\right) .
\end{gathered}
$$

Next, we introduce the column vectors $\mathbf{C}_{n}$

$$
\mathbf{C}_{0}=\left(\mathbf{c}_{0}\right), \quad \mathbf{C}_{ \pm n}=\left(\begin{array}{c}
\mathbf{c}_{ \pm 3 n} \\
\mathbf{c}_{ \pm(3 n-1)} \\
\mathbf{c}_{ \pm(3 n-2)}
\end{array}\right), \quad n>1,
$$

with the property $\mathbf{C}_{-1}(\omega)=\mathbf{C}_{1}^{*}(-\omega)$ so that Eq. (C2) may be rewritten in terms of the solvable matrix three-term recurrence relations

$$
\begin{array}{ll}
\mathbf{Q}_{1}^{-} \mathbf{C}_{0}+\mathbf{Q}_{1} \mathbf{C}_{1}+\mathbf{Q}_{1}^{+} \mathbf{C}_{2}=-\mathbf{F} \mathbf{C}_{1}^{*} \quad(n=1), \\
\mathbf{Q}_{n}^{-} \mathbf{C}_{n-1}+\mathbf{Q}_{n} \mathbf{C}_{n}+\mathbf{Q}_{n}^{+} \mathbf{C}_{n+1}=0 \quad(n>1) .
\end{array}
$$

The supermatrices $\mathbf{Q}_{n}$ and $\mathbf{Q}_{n}^{ \pm}$are given by 


$$
\begin{aligned}
\mathbf{Q}_{n} & =\left(\begin{array}{ccc}
\mathbf{q}_{3 n} & \mathbf{q}_{3 n}^{-} & 2 b_{1} \mathbf{q}_{2(3 n)}^{-} \\
\mathbf{q}_{3 n-1}^{+} & \mathbf{q}_{3 n-1} & \mathbf{q}_{3 n-1}^{-} \\
2 b_{1} \mathbf{q}_{2(3 n-2)}^{+} & \mathbf{q}_{3 n-2}^{+} & \mathbf{q}_{3 n-2}
\end{array}\right), \\
\mathbf{Q}_{n}^{+} & =\left(\begin{array}{ccc}
3 b_{2} \mathbf{q}_{3(3 n)}^{+} & 2 b_{1} \mathbf{q}_{2(3 n)}^{+} & \mathbf{q}_{3 n}^{+} \\
\mathbf{0} & 3 b_{2} \mathbf{q}_{3(3 n-1)}^{+} & 2 b_{1} \mathbf{q}_{2(3 n-1)}^{+} \\
\mathbf{0} & \mathbf{0} & 3 b_{2} \mathbf{q}_{3(3 n-2)}^{+}
\end{array}\right), \\
\mathbf{Q}_{n}^{-} & =\left(\begin{array}{ccc}
3 b_{2} \mathbf{q}_{3(3 n)}^{-} & \mathbf{0} & \mathbf{0} \\
2 b_{1} \mathbf{q}_{2(3 n-1)}^{-} & 3 b_{2} \mathbf{q}_{3(3 n-1)}^{-} & \mathbf{0} \\
\mathbf{q}_{3 n-2}^{-} & 2 b_{1} \mathbf{q}_{2(3 n-2)}^{-} & 3 b_{2} \mathbf{q}_{3(3 n-2)}^{-}
\end{array}\right),
\end{aligned}
$$

with $\mathbf{Q}_{1}^{-}$and $\mathbf{F}$ defined as

$$
\mathbf{Q}_{1}^{-}=\left(\begin{array}{c}
3 b_{2} \mathbf{q}_{9}^{-} \\
2 b_{1} \mathbf{q}_{4}^{-} \\
\mathbf{q}_{1}^{-}
\end{array}\right),
$$

$$
\begin{aligned}
\mathbf{F} & =\left(\begin{array}{ccc}
\mathbf{0} & \mathbf{0} & \mathbf{0} \\
\mathbf{0} & \mathbf{0} & 3 b_{2} \mathbf{q}_{6}^{-} \mathbf{f} \\
\mathbf{0} & 3 b_{2} \mathbf{q}_{3}^{-} \mathbf{f} & 2 b_{1} \mathbf{q}_{2}^{-} \mathbf{f}
\end{array}\right), \\
\mathbf{f} & =\left(\begin{array}{ccccc}
\ddots & \vdots & \vdots & \vdots & . \\
\cdots & 0 & 0 & 1 & \cdots \\
\cdots & 0 & 1 & 0 & \cdots \\
\cdots & 1 & 0 & 0 & \cdots \\
\cdot & \vdots & \vdots & \vdots & \ddots
\end{array}\right) .
\end{aligned}
$$

The matrix $\mathbf{f}$ in the supermatrix $\mathbf{F}$ arises because the distribution function must be real meaning in terms of the column vectors $\mathbf{c}_{n}(\omega)$ that $\mathbf{c}_{-n}(\omega)=\mathbf{c}_{n}^{*}(-\omega)$, which may be rewritten as $\mathbf{c}_{-n}(\omega)=\mathbf{f c}_{n}^{*}(\omega)$. Equations (C3) and (C4) are formally identical to Eqs. (A2) and (A3) above, respectively and their solutions are given in Appendix A.
[1] M. O. Magnasco, Phys. Rev. Lett. 71, 1477 (1993).

[2] S. Scheidl and V. M. Vinokur, Phys. Rev. B 65, 195305 (2002).

[3] P. Hänggi and F. Marchesoni, Rev. Mod. Phys. 81, 387 (2009).

[4] L. Machura, M. Kostur, P. Talkner, J. Luczka, and P. Hänggi, Phys. Rev. E 73, 031105 (2006); P. Hänggi and H. Thomas, Phys. Rep. 88, 207 (1982).

[5] C. C. de Souza Silva, J. Van de Vondel, M. Morelle, and V. V. Moshchalkov, Nature (London) 440, 651 (2006).

[6] D. Suzuki and T. Munakata, Phys. Rev. E 68, 021906 (2003).

[7] W. T. Coffey, Yu. P. Kalmykov, and J. T. Waldron, The Langevin Equation, 2nd ed. (World Scientific, Singapore, 2004).

[8] H. Risken, The Fokker-Planck Equation, 2nd ed. (SpringerVerlag, Berlin, 1989).

[9] W. T. Coffey, Yu. P. Kalmykov, S. V. Titov, and B. P. Mulligan, Phys. Chem. Chem. Phys. 9, 3361 (2007); Phys. Rev. E 75, 041117 (2007); EPL 77, 20011 (2007); J. Phys. A: Math. Theor. 40, F91 (2007).

[10] R. A. Sack, Proc. Phys. Soc. B 70, 402 (1957); R. O. Davies, Physica (Amsterdam) 23, 1067 (1957); W. T. Coffey, S. G. McGoldrick, and K. P. Quinn, Chem. Phys. 125, 99 (1988).

[11] H. C. Brinkman, Physica (Amsterdam) 22, 29 (1956).

[12] L. Machura, M. Kostur, P. Hänggi, P. Talkner, and J. Luczka, Phys. Rev. E 70, 031107 (2004).

[13] D. Zueco and J. L. Garcia-Palacios, Physica E 29, 435 (2005); J. L. Garcia-Palacios and D. Zueco, J. Phys. A 37, 10735 (2004).

[14] S. Denisov, S. Kohler, and P. Hänggi, EPL 85, 40003 (2009); S. Denisov, L. Morales-Molina, S. Flach, and P. Hänggi, Phys. Rev. A 75, 063424 (2007).

[15] J. B. Majer, J. Peguiron, M. Grifoni, M. Tusveld, and J. E. Mooij, Phys. Rev. Lett. 90, 056802 (2003).

[16] H. Linke, T. E. Humphrey, A. Löfgren, and O. Sushkov, Science 286, 2314 (1999).
[17] W. Zwerger, Phys. Rev. B 35, 4737 (1987).

[18] M. P. A. Fisher and W. Zwerger, Phys. Rev. B 32, 6190 (1985).

[19] R. P. Feynman and F. L. Vernon, Ann. Phys. (N. Y.) 24, 118 (1963).

[20] A. O. Caldeira and A. J. Leggett, Physica A 121, 587 (1983); Ann. Phys. (N. Y.) 149, 374 (1983).

[21] E. P. Wigner, Phys. Rev. 40, 749 (1932).

[22] M. Hillery, R. F. O'Connell, M. O. Scully, and E. P. Wigner, Phys. Rep. 106, 121 (1984).

[23] Quantum Mechanics in Phase Space, edited by C. K. Zachos, D. B. Fairlie, and T. L. Curtright (World Scientific, Singapore, 2005).

[24] J. E. Moyal, Proc. Cambridge Philos. Soc. 45, 99 (1949).

[25] R. L. Stratonovich, Zh. Eksp. Teor. Fiz. 31, 1012 (1956); Sov. Phys. JETP 4, 891 (1957).

[26] Yu. P. Kalmykov, W. T. Coffey, and S. V. Titov, J. Stat. Phys. 131, 969 (2008); Phys. Rev. B 77, 104418 (2008); J. Phys. D: Appl. Phys. 41, 134005 (2008); J. Phys. A: Math. Theor. 41, 105302 (2008).

[27] R. Dillenschneider and E. Lutz, Phys. Rev. E 80, 042101 (2009).

[28] J. Ankerhold, P. Pechukas, and H. Grabert, Phys. Rev. Lett. 87, 086802 (2001); Chaos 15, 026106 (2005).

[29] J. Ankerhold, and H. Grabert, Phys. Rev. Lett. 101, 119903(E) (2008).

[30] J. Ankerhold, Quantum Tunnelling in Complex Systems (Springer-Verlag, Berlin, 2007).

[31] U. Weiss, Quantum Dissipative Systems, 3rd ed. (World Scientific, Singapore, 2008).

[32] W. T. Coffey, Yu. P. Kalmykov, S. V. Titov, and L. Cleary, Phys. Rev. E 78, 031114 (2008).

[33] W. T. Coffey, Yu. P. Kalmykov, S. V. Titov, and L. Cleary, Phys. Rev. B 79, 054507 (2009). 
[34] E. P. Wigner, Z. Phys. Chem. Abt. B 19, 203 (1932).

[35] E. Geva, E. Rosenman, and D. Tannor, J. Chem. Phys. 113, 1380 (2000).

[36] H. A. Kramers, Physica (Amsterdam) 7, 284 (1940).

[37] R. L. Stratonovich, Conditional Markov Processes (Moscow University Press, Moscow, 1966).

[38] P. Talkner, M. Campisi, and P. Hänggi, J. Stat. Mech. Theor. Exp. (2009) P02025.

[39] S. Deffner and E. Lutz, e-print arXiv:0902.1858.

[40] M. Kostur and J. Łuczka, Phys. Rev. E 63, 021101 (2001); J. Kula, T. Czernik, and J. Łuczka, Phys. Rev. Lett. 80, 1377 (1998).

[41] S. Chandrasekhar, Rev. Mod. Phys. 15, 1 (1943).

[42] R. E. Wyatt, Quantum Dynamics with Trajectories (Springer,
Berlin, 2005).

[43] W. T. Coffey, Yu. P. Kalmykov, and E. S. Massawe, Phys. Rev. E 48, 77 (1993).

[44] W. T. Coffey, Yu. P. Kalmykov, and J. L. Déjardin, Phys. Rev. B 62, 3480 (2000).

[45] W. T. Coffey and B. V. Paranjape, Proc. R. Ir. Acad., Sect. A 78, 17 (1978).

[46] W. T. Coffey, Adv. Chem. Phys. 63, 69 (1985).

[47] W. T. Coffey, D. S. F. Crothers, and Y. P. Kalmykov, J. NonCryst. Solids 352, 4710 (2006); W. T. Coffey, D. S. F. Crothers, and J. L. Déjardin, Phys. Rev. E 71, 062102 (2005).

[48] S. Shapiro, Phys. Rev. Lett. 11, 80 (1963).

[49] W. T. Coffey, Yu. P. Kalmykov, and J. T. Waldron, Physica A 208, 462 (1994). 